\section{Social media conversations reveal large psychological shifts caused by COVID-19's onset across U.S. cities}

\author{
Ashwini Ashokkumar* and James W. Pennebaker
}

\begin{abstract}
The current research chronicles the unfolding of the early psychological impacts of coronavirus disease 2019 (COVID-19) by analyzing Reddit language from 18 U.S. cities (200,000+ people) and large-scale survey data $(11,000+$ people). Large psychological shifts were found reflecting three distinct phases. When COVID-19 warnings first emerged ("warning phase"), people's attentional focus switched to the impending threat. Anxiety levels surged, and positive emotion and anger dropped. In parallel, people's thinking became more intuitive rather than analytic. When lockdowns began ("isolation phase"), analytic thinking dropped further. People became sadder, and their thinking reflected attempts to process the uncertainty. Familial ties strengthened, but ties to broader social groups weakened. Six weeks after COVID-19's onset ("normalization phase"), people's psychological states stabilized but remained elevated. Most psychological shifts were stronger when the threat of COVID-19 was greater. The magnitude of the observed shifts dwarfed responses to other events that occurred in the previous decade.
\end{abstract}

\section{INTRODUCTION}

The coronavirus disease 2019 (COVID-19) pandemic upended the lives of people across the globe. Following early warnings of an ensuing mental health crisis $(1)$, media reports $(2,3)$ and psychological studies documented some of the psychological effects of the pandemic (4-7), but most relied on small samples or reported cross-sectional findings with only few studies focusing on longitudinal effects $(8,9)$. As a result, we know little about how the social and psychological effects of the pandemic unfolded over time and how the pandemic's impacts compare to past events. Furthermore, almost no studies directly examined the links between the spread of the virus and mental health effects. The current research chronicled the psychological shifts occurring in the 3 months following the onset of the COVID-19 outbreak in the United States to address three research questions. (i) How did changes in people's attention, emotions, thought patterns, and social connections unfold over time? (ii) How were these psychological effects related to the spread of the virus? (iii) How did the psychological impacts of the pandemic compare to events of the past decade? To answer these questions, we analyzed the Reddit conversations of more than 200,000 people across 18 U.S. cities in the months before and after the beginning of the outbreak. Reddit is a popular social media website that hosts city-specific communities in which thousands of people in U.S. cities discuss local issues, which allowed us to obtain reliable estimates of city-specific psychological shifts, including the relationship between the psychological effects and the spread of COVID-19. Furthermore, comparing language produced during the pandemic with language from the preceding decade, we estimated the relative magnitude of the pandemic's impacts. Where possible, the Reddit analysis was complemented by analyzing survey responses of more than 11,000 people in the United States and Canada.

Past studies have identified the temporal effects of short-term upheavals such as hurricanes, earthquakes, and terrorist attacks on people's emotions, cognitive processes, and social connections (10-13).

Department of Psychology, University of Texas Austin, 108 E. Dean Keeton, Austin, TX 78712-0187, USA.

*Corresponding author. Email: ashwinia@utexas.edu
The temporal models generally have focused on how people cope after these collective disasters. For example, the social stage model of coping tracks the changing rates at which people talk to each other about a collective disaster in the weeks following the event (12). Complementary lines of work have examined emotion expressions on social media after disasters $(14,15)$ and how social sharing after a crisis reaps future psychological benefits $(11,16)$. These models assume that disasters are short-lived events that play out within a couple of days and that people are free to come together soon after the event to help them cope in the weeks and months afterward.

Epidemics are a distinct type of upheaval and may produce a unique psychological timeline. Historical accounts of epidemics point to two noteworthy characteristics. First, epidemics are characterized by prolonged uncertainty resulting from several factors. People confront the threat of a new, unknown contagious illness, and simultaneously, a battered economy (17). Adding to the uncertainty, some of the biggest epidemic outbreaks have been characterized by an initial "denialism," wherein governments downplayed the threat of the virus $(18,19)$, producing public disagreement $(20)$, and fostering the spread of misinformation (21). The second notable characteristic of epidemics is prolonged isolation resulting from mandatory or recommended social distancing to reduce contagion. In epidemics, the threat is an invisible pathogen that spreads through human contact, often via asymptomatic individuals, forcing people to actively avoid others. Unlike other upheavals, wherein coming together is an antidote to the collective trauma $(11,13)$, coming together during epidemics increases the risk of spreading the disease. Whereas most upheavals play out within a few days, pandemics last for months or even years, often without a foreseeable end, forcing people to spend an extended time socially isolated from friends and community while knowing little about the current or future course of the disease.

Recent advances in technology have provided an assortment of tools to dynamically and unobtrusively track psychological processes over time. For example, the analysis of social media language has made it possible to study the impact of large-scale events on people's emotions, thought processes, and social behaviors on a scale and temporal horizon that was previously impossible $(11,14,22)$. Adopting this approach, the current research chronicled the temporal changes in 
fundamental psychological processes-attention, emotions, thought patterns, and social connections-as people grappled with the first few months of COVID-19. As depicted in Fig. 1, we assumed that as people became aware of the daunting threat posed by COVID-19, emotional, cognitive, and social changes would surface and, ultimately, interact in recursive ways. Rather than disentangling the interrelationships among these psychological dimensions, our goal was to track their unfolding over time and examine their links with the spread of the virus. Although the pandemic presumably affected several other dimensions (e.g., health behaviors) (23), we focused our analyses on the four dimensions depicted in Fig. 1. These processes have been well documented in the psychological literature on crises and because these dimensions have validated linguistic measures (24). Given that this is one of the first text analysis-based studies of COVID-19, our approach entailed a combination of exploratory and confirmatory analyses. Below, we lay out the theoretical basis for how epidemics might affect the four psychological dimensions.

Research about short-term crises such as earthquakes, hurricanes, and mass shootings has found that people think and talk about a crisis during and immediately after the event $(12,15)$ and that about 6 weeks afterward, most people stop talking about it (12). In contrast, epidemics are relatively long-term crises without a clear end date. Other events such as wars and political uprisings frequently last for extended periods as well, but tracking day-to-day variation in attentional focus for any long-term upheaval has been rare [for an exception, see (25)]. In the first weeks following the onset of COVID-19, people were expected to think and talk about it at high rates. After the first few weeks, as the novelty wore off, we assumed that references to COVID-19 in social media would decline and stabilize at moderate rates. Given that the severity of epidemics varies across time and location, we expected that fluctuations in the spread of the virus would produce parallel changes in attention. In other words, people would likely talk about the virus more when the threat of the virus was greater.

Attending to information about an impending epidemic should produce large-scale emotional effects. The first signs of imminent danger would be expected to produce a surge in people's anxiety $(5,14)$, which should be further exacerbated by the uncertainty resulting from the lack of clear, consistent messages from leaders and experts $(8,26)$. We expected that anxiety levels would remain higher than baseline for a long time because of the continued danger of the virus and prolonged uncertainty (8). Furthermore, a "cabin fever" and loneliness from the lockdowns and isolation may cause feelings of sadness $(4,27)$. Loss of loved ones and exposure to news about death may also produce sadness (4). Governmental failure to control the virus, politicization of epidemic-related issues, and economic depression may also cause people to feel angry (28). Furthermore, recent evidence that feelings of fear and distress were stronger in areas with higher numbers of COVID-19 cases $(5,8)$ suggests that the psychological impacts of COVID-19 would be amplified when the severity of the epidemic is greater. In sum, epidemics were likely to induce high levels of negative emotions (anxiety, sadness, and anger) and low levels of positive emotion, and these effects were expected to be amplified following greater spread of the virus.

Dealing with the uncertainty produced by an epidemic was expected to affect the ways people think. The increase in threat and anxiety has the potential to deplete people's cognitive resources, taxing their cognitive "bandwidth" and working memory (29-31) and as a result, their thinking should switch from being logical and analytic to relying on more "hot" and immediate decision-making shortcuts (32), akin to Kahneman's System 1 thinking (33). A novel threat also forces people to think about and try to make sense of the threat. Studies show that when people are undergoing a trauma or personal problem, they try to process their experience to come to terms with it (34). People frequently ask, "Why is this occurring?" "What can I do?" "When will it be over?" $(35,36)$. In sum, people's thinking was expected to become less analytic and reflect attempts to make sense of the uncertainty, and these cognitive effects would likely be stronger when the threat of the virus is greater.

Almost by definition, COVID-19, like other pandemics, was expected to affect social connections with the community. When faced with the threat of an upheaval, people typically come together to cope and comfort each other (37). Some note the emergence of a "collective resilience" (38) and "catastrophe compassion" (39), which cause people to engage in acts of altruism. In contrast, epidemics discourage people coming together because of the risk of infection. Fears and stigma associated with infection (40) and disagreement regarding the seriousness of the crisis (41) could also trigger mistrust of others and dampen people's sense of community. More important, continued separation from others in the community may increase loneliness $(42,43)$ and weaken people's relationships. At the same time, unlike previous pandemics, the COVID-19 outbreak occurred in the wake of the digital revolution that has allowed for unlimited social interactions through video, audio, and other electronic means, which may have compensated for the decreased face-to-face interactions. In sum, the psychological literature on disasters does not yield clear predictions regarding how epidemics affect social connections.

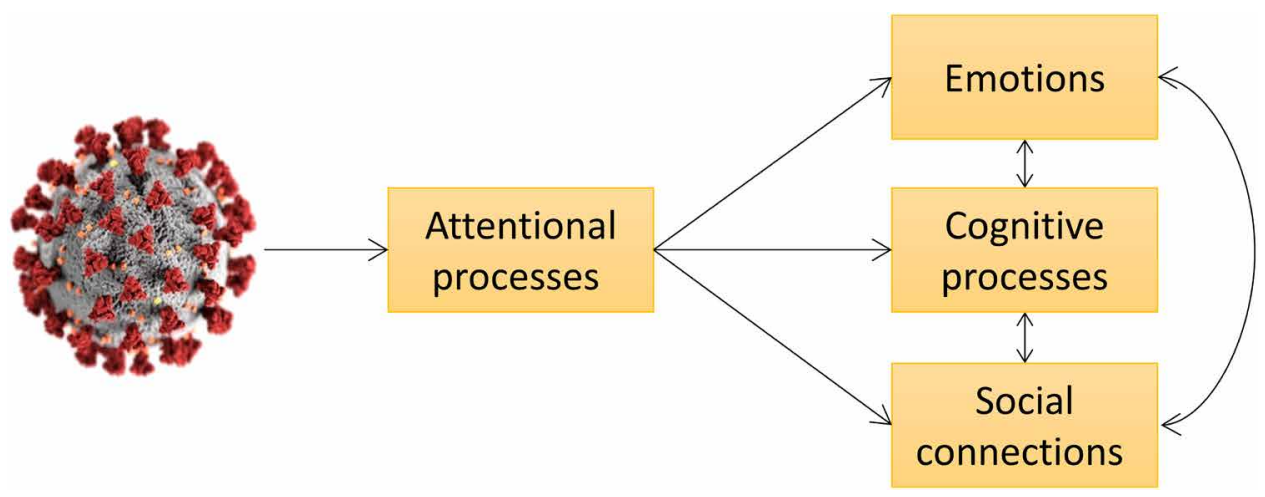

Fig. 1. The interplay of attentional, emotional, cognitive, and social processes during epidemics. 


\section{Overview of the current research}

To study people's reactions to COVID-19, we analyzed the Reddit conversations occurring across 18 U.S. cities. Reddit is a popular social media website that hosts thousands of online communities (or subreddits) in which people publicly discuss various topics of interest. Of relevance here are Reddit's city-specific subreddits meant for people to talk about shared experiences and concerns within their shared geographical space, including traffic, crime, restaurants, and local politics. City subreddits reflect the daily issues and concerns of thousands of people living in identifiable geographic areas, providing for a rich source of information about the psychological states of the people in these cities. Furthermore, because city subreddits are location specific, they allow us to identify links between psychological patterns and city-specific spread of the COVID-19 virus.

In this research, we analyzed the Reddit conversation occurring across 18 city subreddits in the months preceding and following the onset of the COVID-19 crisis and compared these to conversations that occurred in the same cities and during the same time period in 2019. About 1.8 million comments posted by $210,000+$ people on 18 large city Reddit communities between January 10 and May 23 in 2020 were extracted and compared to 1.1 million comments posted by $160,000+$ people in the same communities in 2019 . The psychological dimensions of interest were captured from these Reddit comments and tracked over the time in 2020 and 2019. Second, to capture the link between the severity of the crisis and the psychological impacts, we correlated the psychological dimensions measured from language with the growth rate of the COVID-19 virus. Last, to compare the pandemic's effects with the past decade, we examined 19 million comments posted by $975,000+$ people in the same 18 communities since January 2011.

The focal language analyses were supplemented by data from a large-scale online survey that was completed by 11,140 individuals from the United States and Canada between March 19 and May 23. The survey asked about participants' daily social lives, mental health, and beliefs about the pandemic. The survey sample was composed of two subsamples: (i) a snowball convenience sample obtained by circulating a public website (http://utpsyc.org/covid19/; $n=8848$ ) via the authors' networks and (ii) a crowdsourcing sample, obtained through the company Prolific $(n=2292)$. The snowball convenience sample had a disproportionate number of women and white participants (see Methods), but the Prolific sample was less skewed. Below, we report analyses conducted on the combined sample; but to address concerns regarding generalizability, we report only effects that separately replicated in the relatively more representative Prolific sample (see section S13). Note that we examined data until May 23 because the Black Lives Matter protests following the killing of George Floyd began in the last week of May 2020. More details about both samples are provided in Methods.

In line with Fig. 1, four types of dimensions were examined. Each psychological dimension was measured from people's Reddit conversations using the language analysis software LIWC2015 (24). LIWC is a popular dictionary-based computer text analysis program that provides validated dictionaries for central psychological dimensions and is widely used to analyze social media language.

\section{Attention}

Among the Reddit communities, attention to COVID-19 was operationalized by the mere mention of COVID-19 ("COVID," "coronavirus," and "pandemic"). We determined the percentage of people in the sample who made references to COVID-19 on each day. It was predicted that COVID-19 references would increase rapidly in the first weeks of the outbreak and that attention paid to COVID-19 would stabilize about 6 to 8 weeks later, when the novelty of the crisis presumably wore off. People were expected to refer to COVID-19 more when the threat of the virus was greater.

\section{Emotion}

As explained earlier, the onset of the COVID-19 crisis was expected to produce an increase in expressions of anxiety, sadness, and anger and, by extension, a decrease in positive emotion. These emotional effects were expected to be amplified when the virus spread faster. The four types of emotions (anxiety, anger, sadness, and positive emotion) were measured using validated LIWC2015 dictionaries made up of words associated with each emotion. Example words are provided in table S1 in the Supplementary Materials.

\section{Cognition}

We expected people's thinking to (i) become less analytic and more immediate and (ii) reflect their attempts to better understand and process the issues they were facing. Analytic thinking reflects formal, logical, and hierarchical language measured using a factor analytically derived algorithm of function words made up of high rates of articles and prepositions and low rates of pronouns, auxiliary verbs, negations, conjunctions, and nonreferential adverbs $(44,45)$. Cognitive processing was measured using words associated with causation (e.g., "because"), self-reflection (e.g., "understand"), uncertainty ("maybe"), and so on $(35,46)$. The predicted effects on cognition (drop in analytic thinking, increase in cognitive processing) were expected to be amplified following days when the growth rate of COVID-19 infections was higher.

\section{Social connections}

We did not have clear predictions for the changes in social connections. One argument was that COVID-19, like other collective crises, would bring people together. Alternatively, COVID-19 could undermine social ties because of the inherent dangers of being exposed to the virus by being around others. Social connection was measured from survey responses and Reddit conversations referring to four common social groups - friends, family, city, and country. In addition to using LIWC2015 dictionaries for measuring references to family and friends, we created custom dictionaries to capture references to city and country. Given that the effects on social connections were difficult to predict, analyses were exploratory.

Each psychological dimension was tracked in Reddit across the 18 U.S. cities to determine the temporal language shifts that occurred during the first 3 months of the pandemic. As depicted in Fig. 2, we divided the 2020 timeline (January to May 2020) into four phases on the basis of major COVID-19-related events that occurred in the United States and past research findings:

1) Baseline (January 10 to February 24)

2) Warning phase (February 25 to March 10) when warning signs of an imminent crisis began to emerge (e.g., stock market crash, first U.S. COVID-19-caused death)

3) Isolation phase (March 11 to April 6) beginning around the time when COVID-19 was declared a global pandemic by the World Health Organization and a U.S. national emergency. In this period, several states announced lockdown orders, urging people to self-isolate. Businesses and other organizations closed in-person operations. Daily mobility across the country dropped by more than $40 \%$ (47).

4) Normalization phase (April 7 to May 23) beginning about 6 weeks after the first warnings. Past studies suggest that following an unexpected crisis, the initial surge in interest subsides about 6 weeks 


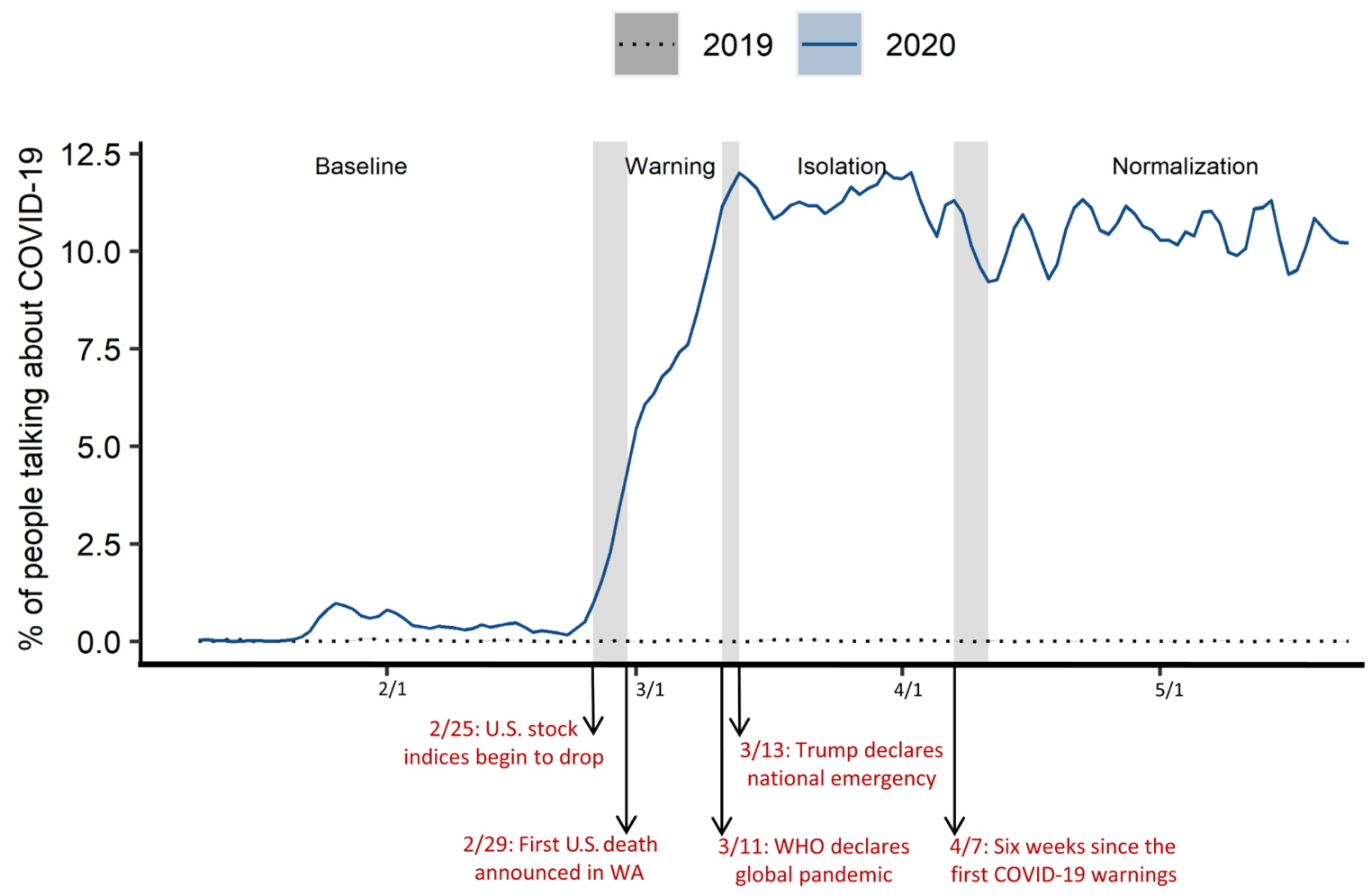

Fig. 2. Three-day rolling means of the percentage of people who mentioned COVID-19 in their posts between January and May 2020 compared with the same months in 2019. The vertical bands delimit the four temporal phases: baseline, warning, isolation, and normalization. Error bands indicate $95 \%$ bootstrapped confidence intervals. The bootstrapped confidence intervals are narrow, which makes the error bands hard to see.

after the event. Although people presumably remained interested in discussing the ongoing COVID-19 pandemic even after the first 6 weeks, the initial rapid surge in attention may have slowed down. In parallel, several policy and cultural changes that had initially received a lot of attention (e.g., social distancing, remote working) became relatively routine for most people, indicating that a new normal was setting in.

Temporal changes were captured over these four phases and compared to the same months in the 2019. Furthermore, daily language scores were correlated with city level and national COVID-19 case growth rates to determine how the psychological effects varied as a function of the spread of the virus. We additionally provide a big-picture perspective by comparing the first 3 months of the COVID-19 outbreak (March to May 2020) to the past decade (2011 to 2020) to determine the magnitude of COVID-19's impact relative to past events. Last, survey data were used when possible, to supplement the Reddit analyses.

\section{RESULTS}

\section{How did the psychological impacts of COVID-19 unfold in the first few months?}

For each person in the focal Reddit dataset (January to May 2019 and 2020), we computed daily means for each language dimension across all their daily comments. To account for variations in the sizes of the city subreddits and avoid overweighting the popular subreddits, we conducted all the analyses on stratified bootstrapped samples with equal-sized subsamples per city. Specifically, we obtained 500 stratified bootstraps each containing 10,000 data points per city per year in 2019 and 2020 (360,000 data points across 18 cities and 2 years in each bootstrapped sample). To deal with the repeated measures nature of the data (because users could post on multiple days), the analyses used mixed effects models that account for random intercepts for each user. The graphs in the article depict bootstrapped means and confidence intervals, and the statistics entail bootstrapped estimates. Note that the effects remain unaltered without bootstrapping (see section S9). All effects reported in the article are significant at $P<0.001$ unless otherwise specified.

As depicted in Fig. 2, the COVID-19 virus became part of people's attentional focus in the United States in the last week of February 2020, when warning signs of an imminent crisis first emerged. During the warning phase, people increasingly talked about the virus in their Reddit conversations, with approximately $5.8 \%$ of people posting about COVID-19 daily on average. People's references to the virus in their conversations peaked during the isolation phase, with a daily average of $11.4 \%$ of the people posting about the pandemic. About 6 weeks after the first warnings, in the first week of April, conversations about the virus stabilized but remained elevated relative to baseline (10.4\% of the sample posted about COVID-19 on average).

To assess the effects of COVID-19 on people's emotions, we examined LIWC scores corresponding to anxiety, sadness, anger, and positive emotion. As shown in Fig. 3 (A to D), average daily emotion scores in 2019 and 2020 were compared across the four temporal phases: baseline, warning, isolation, and normalization. There were clear differences in anxiety expression across the four periods ( $F$ statistics for all the dimensions are reported in section S4). A surge in anxiety immediately followed the emergence of COVID-19 warnings in the last 

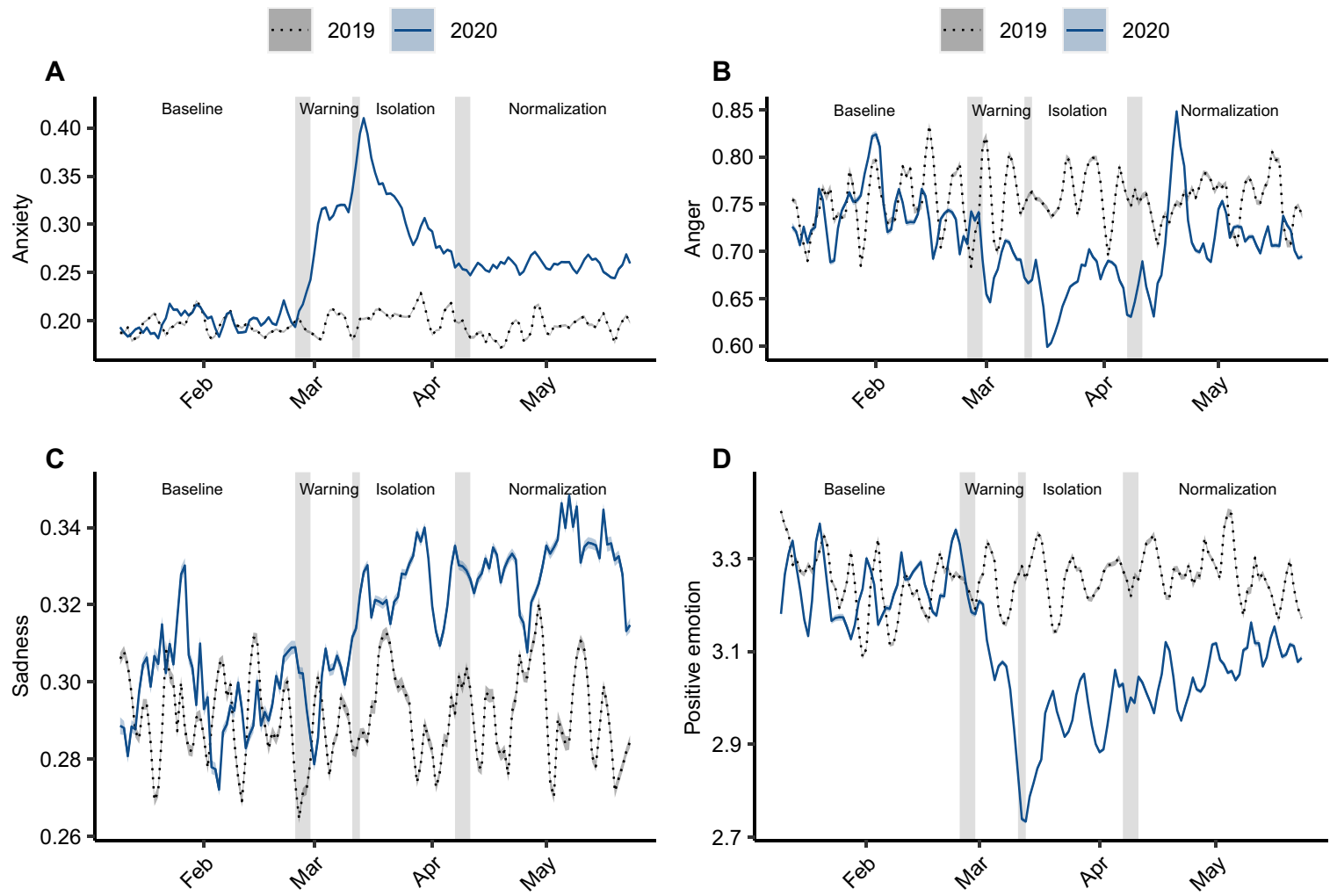

Fig. 3. Changes in emotion produced by the onset of the pandemic. Three-day rolling means of the percentage of anxiety (A), anger (B), sadness (C), and positive emotion (D) words in Reddit posts from 2020 and 2019. The vertical bands delimit the four temporal phases: baseline, warning, isolation, and normalization. Error bands indicate $95 \%$ bootstrapped confidence intervals. The bootstrapped confidence intervals are narrow, which makes the error bands hard to see.

week of February $\left(d_{\text {warning }}=0.10\right)$. Anxiety levels spiked again reaching twice the baseline levels in mid-March when sheltering in place began $\left(d_{\text {isolation }}=0.15\right)$. Effects identified in these big data studies are often small compared to traditional laboratory studies, making them difficult to interpret. On the basis of studies showing that people typically speak about 16,000 words per day in daily conversations (48), the reported anxiety word effect would translate to an increase from about 32 anxiety words per day at baseline to about 64 words at the peak in March. Although anxiety dropped in the normalization period, it remained elevated relative to baseline $\left(d_{\text {normalization }}=\right.$ 0.07 ), consistent with recent findings based on Twitter language (43). Analysis of survey data suggested that participants were most anxious about the health of their family members and about inadvertently spreading the virus to strangers, followed by concerns about getting sick and dying themselves (see section S13).

As expected, sadness increased, and positive emotion decreased. The increase in sadness was relatively small and gradual, remaining elevated for months starting in the isolation phase $\left(d_{\text {isolation }}=0.03\right.$; $d_{\text {normalization }}=0.04$ ). The frequency of sadness words increased by approximately $11 \%$ in the normalization phase relative to baseline. As can be seen in Fig. 3D, the positive emotion shifts mirrored anxiety patterns but in the opposite direction, dropping first with the emergence of warning signals $\left(d_{\text {warning }}=-0.04\right)$ and reaching its lowest point in mid-March, which amounted to a drop of about $10 \%$ in the isolation phase relative to baseline $\left(d_{\text {isolation }}=-0.11\right)$, and remaining diminished in the normalization period $\left(d_{\text {isolation }}=-0.06\right)$. Diverging from our prediction that anger would increase, anger expressions dropped following the outbreak $\left(d_{\text {isolation }}=-0.05\right)$, which is unlike the pattern seen after man-made crises such as terrorist attacks and mass shootings (10). Note that the increase in anger in mid-April corresponds to the anti-lockdown demonstrations organized across U.S. cities. Notably, 3 months after the beginning of the pandemic, people's anxiety and sadness remained elevated, and positive emotion remained diminished. The Supplementary Materials includes graphs comparing the effects across the 18 cities (section S12).

The language analyses using LIWC also revealed large changes in people's thinking styles. As shown in Fig. 4, being surrounded by the threat and uncertainty produced by COVID-19 warning signals was associated with drops in analytic thinking $\left(d_{\text {warning }}=-0.05\right)$ relative to baseline and to the same period in 2019. Analytic thinking dropped further, reaching its lowest point in March when most people began to socially isolate themselves $\left(d_{\text {isolation }}=-0.16\right)$ and remained at suppressed levels for months $\left(d_{\text {normalization }}=-0.11\right)$, indicating that the pandemic pushed people to think in a more immediate and less analytic manner. The findings are consistent with research finding that upheavals that upend people's personal lives reduce analytic thinking (32) and disrupt related behaviors such as academic performance (49).

We also investigated whether people's thinking reflected attempts to make sense of and process what was happening by tracking cognitive processing words such as think, believe, and because in their conversations. As illustrated in Fig. 4B, people began to use higher rates of cognitive processing words when COVID-19 warning signals began $\left(d_{\text {warning }}=0.03\right)$, reaching the highest point when they 

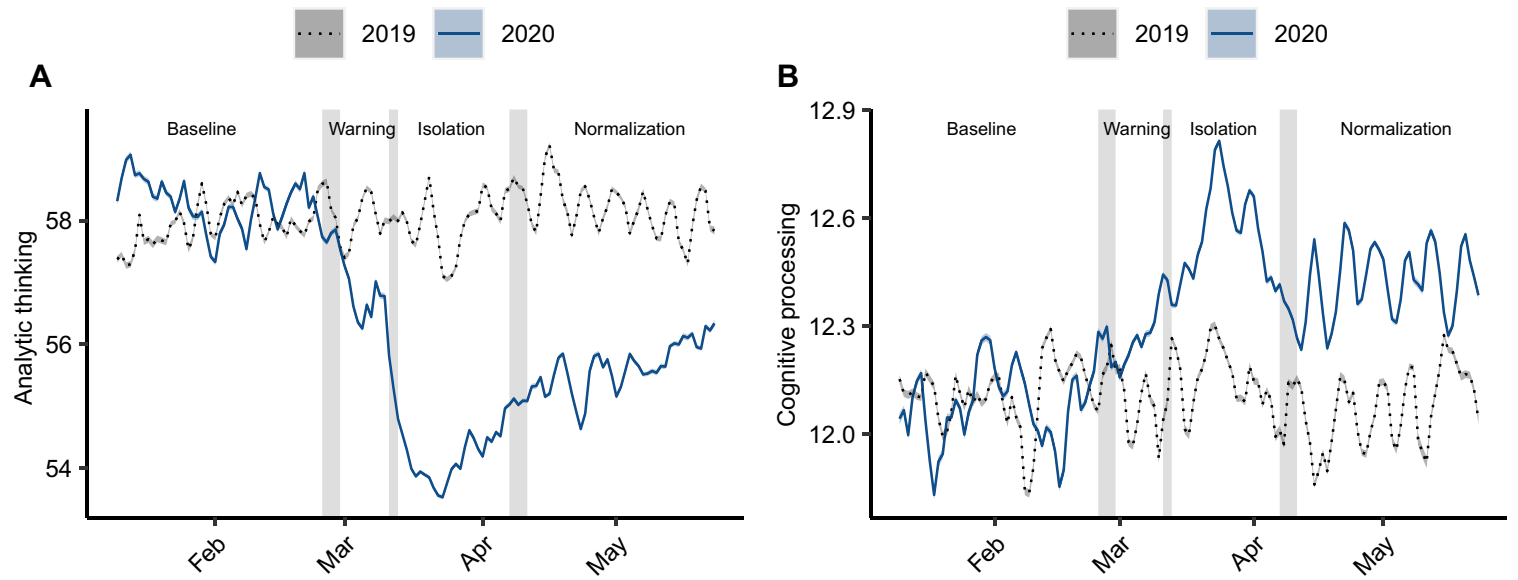

Fig. 4. Changes in cognition produced by the onset of the pandemic. Three-day rolling means of analytic thinking scores (A) and the percentage of cognitive processing words (B) in Reddit posts from 2020 and 2019. The vertical bands delimit the four temporal phases: baseline, warning, isolation, and normalization. Error bands indicate $95 \%$ bootstrapped confidence intervals. The bootstrapped confidence intervals are narrow, which makes the error bands hard to see.

were confined to their homes $\left(d_{\text {isolation }}=0.08\right)$ and remaining elevated in the normalization period $\left(d_{\text {normalization }}=0.06\right)$. People's use of cognitive processing words increased by approximately $4 \%$ in the isolation phase, and given the high base rate of cognitive processing word use (about $12 \%$ of words at baseline), the $4 \%$ rise would amount to a substantial increase in terms of raw word frequency. The surge in cognitive processing is a common consequence of collective upheavals (10), but unlike most crises, cognitive processing remained high even months after the outbreak, indicating that people were continuing to face and work through various issues for an extended period of time.

Changes in people's social connections and sense of community were also found. Survey participants self-reported that COVID-19 changed their connections with four of their social groups-family, friends, city, and country. On five-point scales in which values above and below the midpoint (i.e., 3) indicated increases and decreases in connection, respectively, people reported that the outbreak had made them feel more connected with their families $(d=0.26$; relative to the scale's midpoint) but less connected with the three other groups: their friends $(d=-0.38)$, city $(d=-0.51)$, and country $(d=-0.29)$ (see Fig. 5).

Tracking how much people focused on each of the four social groups in their Reddit conversations revealed parallel findings. We assumed that people think and talk most about the groups that they are most concerned with. As depicted in Fig. 5B, after the COVID-19 outbreak, people's references to their families increased $\left(d_{\text {isolation }}=0.07\right)$. At the same time, their focus on their friends dropped $\left(d_{\text {isolation }}=-0.03\right.$, $P=0.009)$, as did their references to their cities $\left(d_{\text {isolation }}=-0.19\right)$. As seen in fig. S1 in the Supplementary Materials, in the isolation phase, the use of family words increased by about $27 \%$, and friends and city words dropped by 8 and 37\%, respectively. Country-related references on Reddit increased, but these references were driven by the use of impersonal references to the United States common in COVID-19-related news (e.g., "the US," "United States"; $d_{\text {isolation }}=0.02$, $P=0.008$ ) rather than more personal references (e.g., "America," "our nation"; $d_{\text {isolation }}=0.0006 ; P=0.24$ ) (see section S11 for more details). It is possible that the references to the United States reflect increases in discussions about the COVID-19 crisis rather than feelings of closeness. The survey and Reddit findings together suggest that the COVID-19 crisis brought people closer to their families but weakened their relationships with the broader community outside of their homes, including their friends. Exploratory analysis of survey ratings suggest that people's sense of community may have weakened further as the crisis progressed (see section S13).

\section{Robustness checks}

We conducted several robustness tests for the main Reddit analyses. To rule out cohort effects, we conducted within-participants analysis of only Reddit users who posted both before and during COVID-19 (see section S5), and the findings remained strong. Furthermore, we compared the effects across the 18 cities (see section S12) and found the psychological timeline to be comparable across the cities. Furthermore, we analyzed only posts that did not mention COVID-19 and found that the effects are not limited to interactions about the pandemic (see section S6). In addition, testing whether the effects are driven by the proportion of people who excessively talked about the pandemic (section S7), we found parallel effects for people who discussed the pandemic and those who never did once. In section S9, we also demonstrate that weighting means, as opposed to bootstrapping, results in the same results. We also report exploratory analyses assessing the extent to which the observed effects spill over into other domains of people's lives such as when they are interacting in topic-specific communities (e.g., gaming, movies; see section S8). The results revealed that the emotional effects carried over to noncity subreddits, but most cognitive and social effects did not. Perhaps becoming immersed in specific contexts (e.g., sports or gaming) provides a respite from the cognitive and social consequences of the pandemic. However, these analyses are not conclusive because there are variations in the specific noncity subreddits that people visited before versus during the pandemic, potentially confounding the analysis.

\section{Did greater spread of the COVID-19 virus amplify the psychological impacts?}

To examine the link between the spread of the virus and people's psychological states, we conducted exploratory analyses testing whether daily growth rates of infections predicted the observed psychological shifts on the following day. The growth rate of infections, as opposed to deaths, was examined because only few deaths occurred in some of the cities during the early days of the pandemic. Daily cumulative 

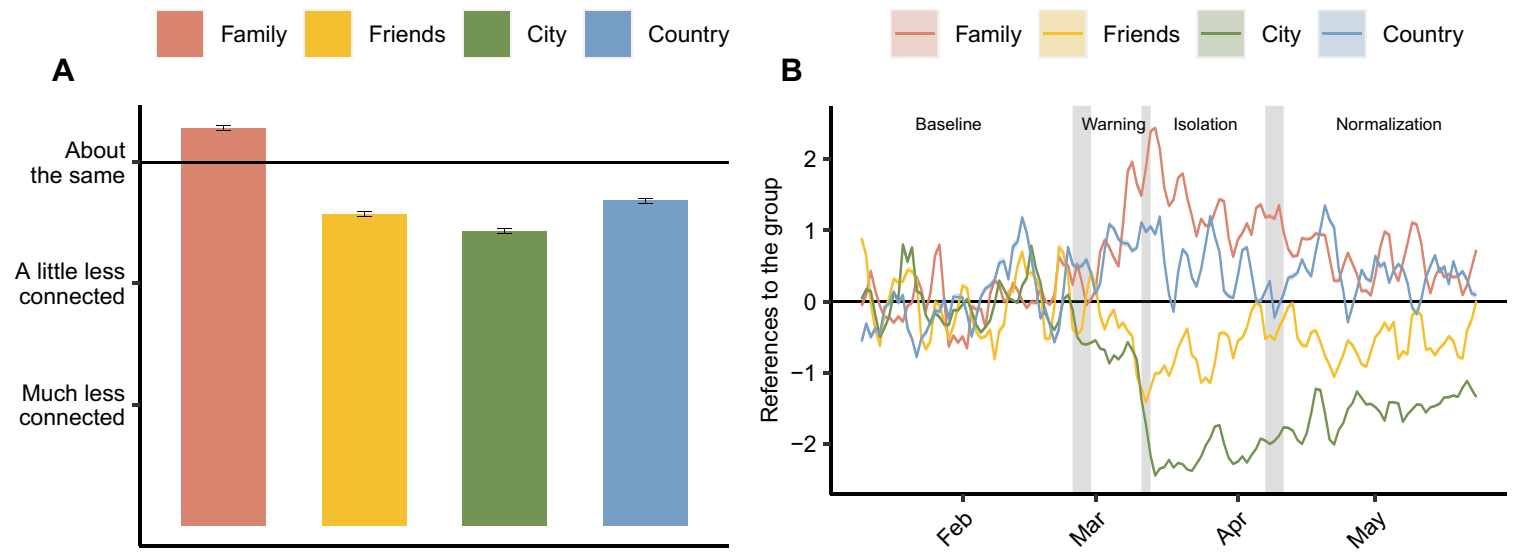

Fig. 5. Changes in social connections produced by the onset of the pandemic. Survey ratings regarding how much COVID-19 increased or decreased people's feelings of connection with various social groups (A) and three-day rolling means of normalized scores indicating how much people referred to each of the social groups on Reddit (B). The vertical bands delimit the four temporal phases: baseline, warning, isolation, and normalization. Error bars (A) indicate $95 \%$ confidence intervals, and error bands (B) indicate $95 \%$ bootstrapped confidence intervals. The bootstrapped confidence intervals are narrow, which makes the error bands hard to see.

COVID-19 infection counts at the county and national levels were obtained from the New York Times COVID-19 case count database. In the analyses reported below, growth rate was computed assuming an exponential model of disease spread using the formula $\ln \left(I_{t} / I_{t-1}\right)$, where $I$ is the cumulative number of infections (in a city or in the United States) and $t$ is the time (day) (50). Growth rate was lagged by 1 day because it presumably takes a day for information regarding the number of cases to reach the public. Lagged daily growth rate was then used to predict city-level daily means of the language dimensions. The resulting city-level dataset had 2430 daily means of language dimensions and daily national- and city-level growth rates. In each model, the national- and city-level growth rates were entered as predictors and a standardized psychological dimension (e.g., anxiety) was the outcome. The models tested the extent to which city- and national-level growth rates predicted shifts in the focal psychological dimensions. In section S10, we report the same analyses assuming a linear growth model, but exponential growth rate generally accounted for more variance $\left[\mathrm{R}^{2}\right.$ (coefficient of determination)] across the psychological dimensions.

National- and city-level growth rates amplified several of the examined psychological effects such that the effects were more pronounced following days when the virus spread faster. First, as shown in Fig. 6, tracking the percentage of COVID-19 references in people's posts, we found that people talked about COVID-19 more following days that witnessed higher national- and city-level infection growth rates of the virus [city level: $b=2.08, t(2391)=15.83$; national level: $b=0.59, t(2391)=3.89]$.

To test whether the emotional shifts were linked with the spread of the virus, we examined whether national and cities' daily growth rates of COVID-19 infections predicted average city-level emotion scores on the following day. As shown in Fig. 6, we found independent effects of national- and city-level infection growth rates such that greater spread of the virus at both the city and national levels was followed by higher anxiety [city level: $b=1.84, t(2391)=14.20$; national level: $b=1.36, t(2391)=9.06$ ] and lower positive emotion [city level: $b=-1.09, t(2391)=-7.96$; national level: $b=-0.66, t(2391)=-4.15]$ on the following day. We found a weak effect of city level infection growth rate on anger and no effect for sadness (see section S10).
Furthermore, the cognitive dimensions were particularly associated with city-level infection growth rate. Analytic thinking dropped more following days with a higher growth rate of infections, and this effect was stronger for city-level growth rate [city level: $b=-1.70, t(2391)=-12.60$; national level: $b=-0.37, t(2391)=-2.37, P=0.02]$. In the same vein, the increase in cognitive processing was more pronounced following days when the infection growth rate was higher at the city level but not the national level, city level: $b=1.02, t(2391=7.34$; national level: $b=-0.04, t(2391)=-0.22, P=0.82$ (see Fig. 6). Notably, city-level growth rate had a greater effect on cognitive patterns than national-level growth rate, marking a link between immediate COVID-19 threat and cognitive patterns.

The growth rate of the virus was also associated with some of the social dimensions. People talked more about their family following days when the virus spread faster in their city or the country [city level: $b=0.83, t(2391)=5.94$; national level: $b=0.61, t(2391)=-3.79]$. Higher city-level growth rate was associated with lower likelihood of conversations about one's city [city level: $b=-1.44, t(2391)=-10.53$; national level: $b=-0.34, t(2391)=-2.14, P=0.03]$, perhaps reflecting the fact that people stayed home more when the threat of the virus was greater locally. There was also a weak negative effect of city-level growth rate on talking about friends [city level: $b=-0.41$, $t(2391)=-2.95, P=0.003$; national level: $b=-0.09, t(2391)=-0.58$, $P=0.56]$.

City and national growth rates had comparable effects on emotion dimensions (higher anxiety and lower positive emotion when city or national growth rate was higher), but city growth rate had a particularly stronger effect on attention- and cognition-related dimensions (more references to COVID-19, lower analytic thinking, and higher cognitive processing when city growth rate was higher). Apparently, emotions were shaped by the severity of not only local but also national crisis, perhaps indicating that exposure to the wide news coverage of the national crisis affected people's emotion almost as much as local threat did. In contrast, attention and cognitive effects primarily reflected the immediacy of local crisis rather than more distal threat. Given that these were exploratory analyses, we leave it to future research to further explore this issue. 


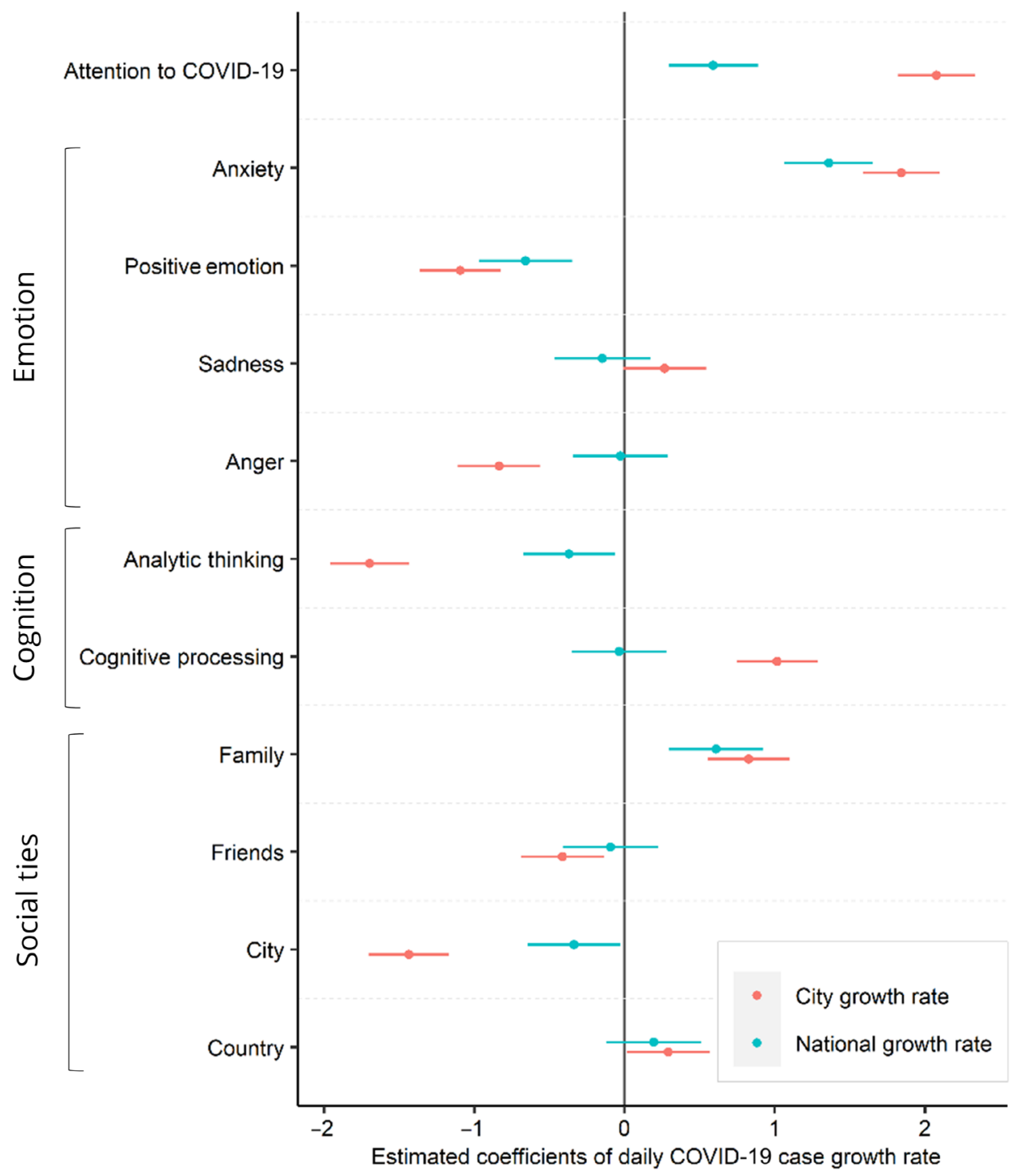

Fig. 6. Estimated coefficients of the effect of daily COVID-19 case growth rates at the city and national levels on aggregated city-level psychological dimensions on the following day. Positive (negative) coefficients indicate a positive (negative) association between virus spread and the corresponding psychological dimension.

\section{How do the psychological impacts of COVID-19 compare with the past decade?}

Last, it is helpful to provide a big picture perspective of the social and psychological impact of COVID-19 by comparing responses to the pandemic with events of the past decade. Accordingly, 19,911,103 comments posted by 975,286 people on the 18 city subreddits between January 2011 and May 2020 were examined. Weighted monthly means were computed to avoid overweighting popular subreddits. The resulting dataset contained 113 monthly means spanning 2011 to 2020 . Figure 7 depicts the monthly means for all the focal psychological dimensions. Relative to any national events that occurred between 2011 and 2020, the shifts during COVID-19 (March to May 2020) were orders of magnitude greater for almost all the language measures, indicating that the mental health crisis fueled by the COVID-19 pandemic is unlike anything in the recent past of the United States.

\section{DISCUSSION}

Beyond the unprecedented health and economic impacts of COVID-19, the pandemic had large and meaningful social and psychological effects during the first 3 months of its reign in the United States. Relying on community-wide Reddit language and survey data, we see how people's attention, emotions, thoughts patterns, and social ties shifted over time as the crisis unfolded. The psychological effects typically first surfaced and began to rise when COVID-19 warning signals first emerged (the "warning" phase) and peaked in mid-March following stay-at-home orders ("isolation" phase). Most effects stabilized in April but remained elevated through the end of our data collection in late May ("normalization" phase). The psychological effects were amplified when the virus spread faster, as indicated by the associations with national- and city-level growth rates of infection. Furthermore, a comparison of people's language during the pandemic with the previous decade revealed that the mental health 

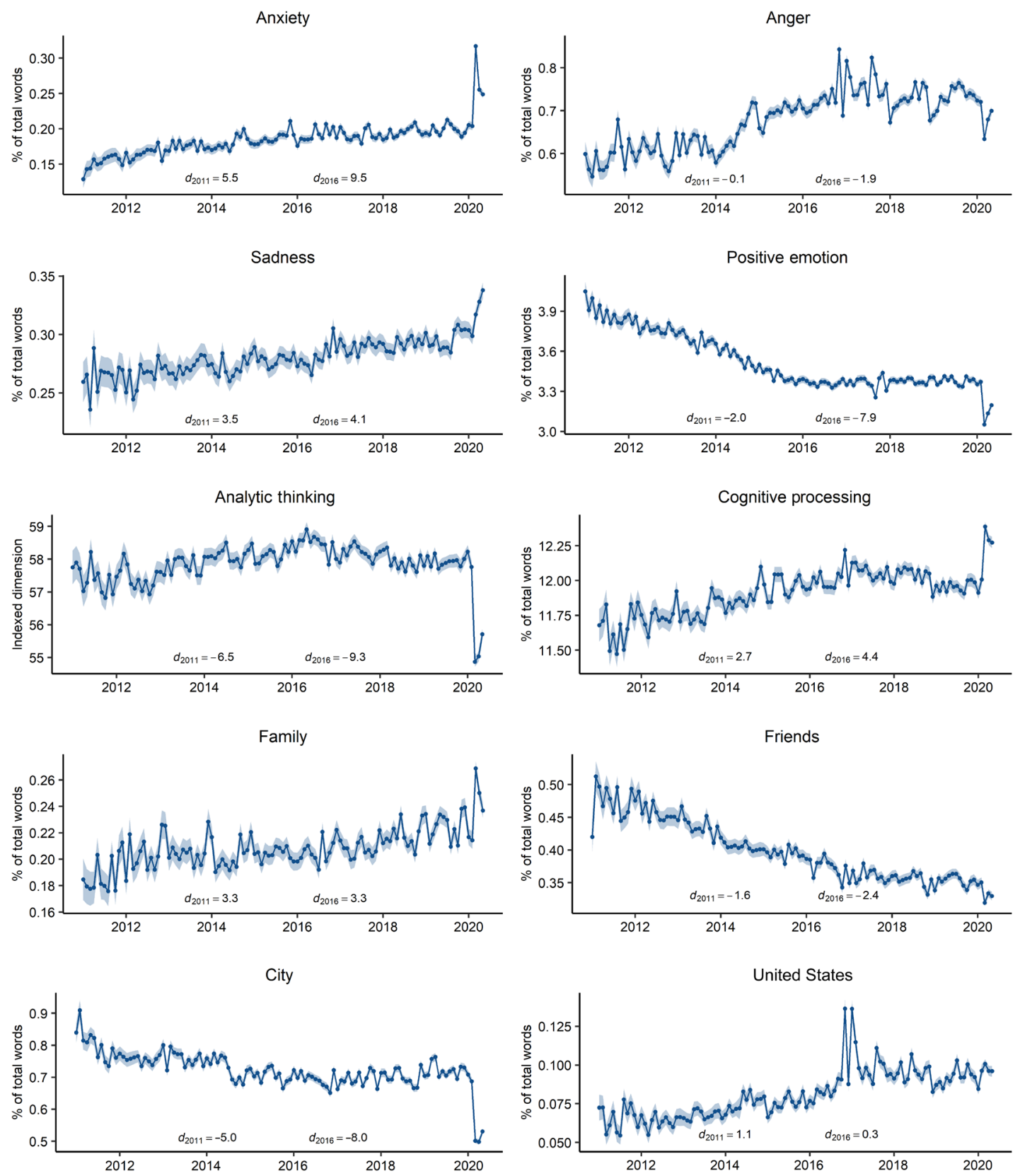

Fig. 7. Weighted monthly averages of language dimensions across 18 city subreddits since 2011. The statistics above the $x$ axes represent effect sizes (Cohen's $d$ ) computed using monthly average scores. $d_{2011}$ represents the difference between the monthly averages during the pandemic (March to May 2020) and the monthly averages in the period from 2011 to February 2020. $d_{2016}$ represents the difference between the monthly averages during the pandemic (March to May 2020) and the monthly averages from 2016 to February 2020.

impacts of COVID-19 may have been greater than anything the United States has seen in recent times.

The uncertainty that the pandemic infused into people's lives produced shifts in their attention, emotions, and thinking styles. References to COVID-19 surged when the first COVID-19 warnings emerged, and in parallel, people's language revealed sharp increases in anxiety and drops in positive emotion. At the same time, analytic thinking was diminished, which previous studies have found to be linked with poor academic performance (45). These effects were amplified when most people began to isolate themselves because of lockdowns. During the isolation phase, language also indicated increases in sadness and in attempts to process and make sense of what was going on. The disruption of emotion and thought patterns are illustrated by the following excerpts from Reddit comments:

"Feelings speculation more feelings more speculation fear fear fear fear fear. Speculations and fear."

"I'm freaking out. I am panicking. Do you know what anxiety is"

"Struggling with anxiety... Terrified of the days when I need to go get groceries." 
"I can't focus on anything. In 2 weeks I've gotten maybe 2 days worth of work done."

"I'm burned out. Mentally exhausted."

Particularly relevant to the pandemic were the disruptive effects on people's social ties in the community. Survey respondents reported feeling closer to family but more distant from broader social groups such as friends, their city, and country. Analyses of the attention people afforded to these groups in their social media conversations largely paralleled the survey results, indicating an increased focus on family and a decreased focus on friends and city, especially during the isolation phase. Conversations focused on the United States showed a small increase, but this was largely driven by increases in impersonal references (e.g., "the US") than personal references ("our nation"), which could simply reflect an increase in United States-related news. Contrary to the popular narrative that collective crises bind people together, the COVID-19 crisis may have disrupted the social fabric other than people's kin networks, as revealed by the below Reddit excerpts. Our data relied on Reddit users and online survey respondents-people familiar with the internet and social media. Somewhat unexpected was that they felt less connected to friends and their community after the crisis starteddespite their having (and using) online social media sources. Lack of trust in the government and increasing polarization during the crisis may also have weakened people's sense of community.

"I WAS ALONE BEFORE, BUT THIS IS JUST TOO HECKIN MUCH. I'M SO TIRED OF BEING ALONE. DANCING ALONE. SLEEPING ALONE... ALONE! ALONE! ALONE!"

"I miss the city man. I miss the energy, the atmosphere. ...I miss the crowds. I miss the people."

"* cries in loneliness because I have no friends or family to hang out with all break"

The effects observed across the 18 cities reflect three social psychological phases corresponding to (i) the initial warning signals indicating the emergence of an epidemic, (ii) the beginning of social isolation, and (iii) the shift toward normalization of life during the epidemic. The isolation phase is unique to epidemics during which people are forced to remain apart. The warning and normalization phases roughly correspond to past models (e.g., emergency and adaptation, respectively, in the social stage model) (12). The primary difference is that in previous single-event upheavals, adaptation referred to returning to baseline levels of behavior. The COVID-19 crisis, and presumably other long-term upheavals, simply settle on a new baseline during the normalization phase. In this period, people's altered psychological states persisted but stabilized, reflecting a new normal. Future research could determine whether the stabilization marks a permanent shift in baseline psychological state.

Particularly promising from a methodology perspective is that, within the Reddit samples, it was possible to detect psychological shifts in naturally occurring conversations in relatively neutral online communities wherein people discuss a range of topics. Examining city communities allowed us to probe the relationship between the severity of the crisis at local and national levels and the observed psychological impacts. The availability of rich records of Reddit conversations also made it possible to go back in time to retrieve clues about the pre-COVID-19 psychological states of people across cities. As is common in social media studies, our effect sizes are smaller than the effects typically reported in controlled laboratory studies that artificially control for most extraneous factors $(51,52)$. Even in the current research, the social connection effects captured in survey data are substantially larger than parallel Reddit effects testing the same relationships. Echoing other scholars, we believe that the big data approach provides reliable representations of real-world effects (53) and that small but reliable effects are most likely to be real and can scale up to produce substantial consequences at national or global levels (52).

The current study has several limitations. The temporal analysis focusing on large U.S. cities is not informative about the unfolding of psychological effects in smaller cities and rural areas and other areas around the world. Furthermore, the current study modeled temporal effects of the pandemic without accounting for how people's emotions or cognitive patterns may have been influenced by the posts of others in the community (e.g., emotional contagion). Also, the studies largely examined the psychological impacts on the average person without probing individual differences. Reports suggesting that the crisis disproportionately affected minority communities underscore the need for future research to delve deeper into demographics- and personality-based differences in psychological effects.

The present study of 200,000+ people across 18 large U.S. cities during the COVID-19 pandemic offers useful insights for both theory development and practice. The findings point to a temporal model for understanding the psychological dynamics of a complex event as it unfolds. To date, most natural and man-made disaster research has focused on discrete events that often had a major impact on a specified group of people over a relatively brief time. Future theorizing must begin to systematically identify longer-term coping patterns. Unlike most previously studied upheavals, COVID-19 was a nonsocial crisis that mandated actively avoiding others. Our findings challenge the widely prevalent notion that crises unite people, highlighting the need for research exploring the complex effects of different types of crises on perceived and actual social networks $(37,54)$.

One interesting by-product of the Reddit language analyses was the ability to see shifts in the ways people were thinking over time. For example, the cognitive processing language pointed to the prolonged cognitive load that people were exhibiting. There is now a burgeoning research literature on the potential disruptions that cognitive load and reduced working memory have on decision-making, academic and work performance, and other important cognitive outcomes (55). Overall, the research underscores the critical need for policy interventions addressing the large-scale psychological disruptions and people's weakened sense of community. Practitioners and organizations should make mental health resources more widely accessible and develop community-building interventions to maintain strong social networks even during isolating circumstances.

\section{METHODS}

The current study analyzed two types of samples. The Reddit sample was an archival dataset of the comments posted on the social media website Reddit, and the survey sample consisted of people who completed a large-scale survey about COVID-19. The research was reviewed by the Institutional Review Board of the authors' institution and deemed as an exempt study (see section S1 for a detailed discussion of ethics).

\section{Reddit}

The current research examined conversations from the popular social media website Reddit. Reddit hosts thousands of online communities that people actively engaged in, including city-based communities. Using the Pushshift API, we extracted all comments posted on 
18 city-related Reddit communities corresponding to large U.S. cities between January 10 and May 23 in 2020 and 2019. For the analysis in the final section, comments posted between January 2011 and May 2020 were extracted. The cities included Atlanta, Austin, Boston, Chicago, Houston, Los Angeles, Orlando, Nashville, New York City, Philadelphia, Phoenix, Portland, Raleigh, San Diego, San Francisco, Seattle, St. Louis, and Washington DC. The city-related subreddits were selected if they had at least 50,000 subscribers. We tried to cover most geographic regions within the United States. An online list of the most popular city subreddits was used as a starting point, but some cities that had more than 50,000 subscribers were excluded (e.g., Dallas, Baltimore) because we had already included other nearby cities (e.g., Houston and Austin, and Washington DC). These decisions were made before data extraction, in a relatively arbitrary manner. The rationale for the dates (January 10 to May 23) was to analyze posts beginning about 10 days after New Year's Eve to ensure that the baseline was unaffected by conversations that occurred during the holiday season. We examined data until May 23 because the Black Lives Matter protests following the killing of George Floyd began in the last week of May 2020.

The raw sample extracted from Reddit had 3,183,306 comments posted by 285,341 people in 2020 and 2,137,984 comments posted by 218,342 people in 2019 . Because extremely short texts would cause the LIWC scores (percentages of total words for each text) to become noisy, comments with fewer than 15 words were excluded. Setting the threshold to 20 or 25 words did not alter the findings. To eliminate bots, usernames that ended with "Bot" or case variations of "_bot" and "-bot" were eliminated. Some other common Reddit bots previously identified in our laboratory were excluded. The final sample had $1,824,767$ posts from 216,811 unique users in 2020 and $1,179,707$ posts from 160,340 unique users in 2019. Although we cannot get information about the demographics of individual users or users of specific subreddits, aggregated demographic information suggest that Reddit's users tend to be male (over 60\%) and younger (64\% users are below age 29). The ethnic distribution of Reddit users follows U.S. population trends, and most Redditors have some college education or a degree.

\section{Survey}

The survey sample included 8848 people $(80.19 \%$ women, $1.3 \%$ nonbinary; $M_{\text {age }}=43.10 ; S D_{\text {age }}=16.31 ; 80.97 \%$ white) who completed the survey on a public website (https://utpsyc.org/COVID19/) and 2292 people $\left(51.81 \%\right.$ women, $1.3 \%$ nonbinary; $M_{\text {age }}=32.95$; $S D_{\text {age }}=11.91 ; 69.89 \%$ white) who were recruited via Prolific between March 19 and May 23. The survey website was circulated via the authors' personal networks and social media, which was essentially a snowball sampling method. People from around the world took the website survey, but only responses from the United States and Canada were used in these analyses. The website sample is not representative (skewed toward women and white people), which is not unusual given that it was a convenience sample. The gender skew has been a common issue with COVID-19-related convenience samples (56) perhaps reflecting that women were more motivated to introspect on and address mental health issues early on in the pandemic. However, the Prolific sample was less skewed and provided for a relatively more representative replication sample. All survey results reported in the paper were replicated in the Prolific sample (see section S13), addressing concerns that the effects are a methodological artifact.

\section{SUPPLEMENTARY MATERIALS}

Supplementary material for this article is available at https://science.org/doi/10.1126/ sciadv.abg7843

\section{REFERENCES AND NOTES}

1. S. Galea, R. M. Merchant, N. Lurie, The mental health consequences of COVID-19 and physical distancing: The need for prevention and early intervention. JAMA Intern. Med. 180, 817-818 (2020).

2. J. Hamblin, Is Everyone Depressed? (The Atlantic, 2020); www.theatlantic.com/health/ archive/2020/05/depression-coronavirus/611986/.

3. F. Manjoo, Opinion |The Hidden 'Fourth Wave' of the Pandemic (N. Y. Times, 2020); www. nytimes.com/2020/12/09/opinion/coronavirus-mental-health.html.

4. R. Rossi, V. Socci, D. Talevi, S. Mensi, C. Niolu, F. Pacitti, A. Di Marco, A. Rossi, A. Siracusano, G. Di Lorenzo, COVID-19 pandemic and lockdown measures impact on mental health among the general population in Italy. Front. Psych. 11, 790 (2020).

5. K. M. Fitzpatrick, C. Harris, G. Drawve, Fear of COVID-19 and the mental health consequences in America. Psychol. Trauma Theory Res. Pract. Policy 12, S17-S21 (2020).

6. D. Fancourt, A. Steptoe, F. Bu, Trajectories of anxiety and depressive symptoms during enforced isolation due to COVID-19 in England: A longitudinal observational study. Lancet Psychiatry 8, 141-149 (2021).

7. M. Luchetti, J. H. Lee, D. Aschwanden, A. Sesker, J. E. Strickhouser, A. Terracciano, A. R. Sutin, The trajectory of loneliness in response to COVID-19. Am. Psychol. 75, 897-908 (2020).

8. E. A. Holman, R. R. Thompson, D. R. Garfin, R. C. Silver, The unfolding COVID-19 pandemic: A probability-based, nationally representative study of mental health in the United States. Sci. Adv. 6, eabd5390 (2020)

9. M. T. Hawes, A. K. Szenczy, T. M. Olino, B. D. Nelson, D. N. Klein, Trajectories of depression, anxiety and pandemic experiences; A longitudinal study of youth in New York during the Spring-Summer of 2020. Psychiatry Res. 298, 113778 (2021).

10. M. A. Cohn, M. R. Mehl, J. W. Pennebaker, Linguistic markers of psychological change surrounding September 11, 2001. Psychol. Sci. 15, 687-693 (2004).

11. D. Garcia, B. Rimé, Collective emotions and social resilience in the digital traces after a terrorist attack. Psychol. Sci. 30, 617-628 (2019).

12. J. W. Pennebaker, K. D. Harber, A social stage model of collective coping: The Loma Prieta Earthquake and The Persian Gulf War. J. Soc. Issues 49, 125-145 (1993).

13. R. C. Silver, E. A. Holman, D. N. McIntosh, M. Poulin, V. Gil-Rivas, Nationwide longitudinal study of psychological responses to September 11. JAMA 288, 1235-1244 (2002).

14. N. M. Jones, R. C. Silver, This is not a drill: Anxiety on Twitter following the 2018 Hawaii false missile alert. Am. Psychol. 75, 683-693 (2020).

15. N. M. Jones, S. P. Wojcik, J. Sweeting, R. C. Silver, Tweeting negative emotion: An investigation of Twitter data in the aftermath of violence on college campuses. Psychol. Methods 21, 526-541 (2016).

16. B. Rimé, D. Páez, N. Basabe, F. Martínez, Social sharing of emotion, post-traumatic growth, and emotional climate: Follow-up of Spanish citizen's response to the collective trauma of March 11th terrorist attacks in Madrid. Eur. J. Soc. Psychol. 40, 1029-1045 (2010).

17. R. Kochhar, Unemployment Rose Higher in Three Months of COVID-19 Than it did in Two Years of the Great Recession (Pew Research Center, 2020); www.pewresearch.org/ fact-tank/2020/06/11/unemployment-rose-higher-in-three-months-of-covid-19-than-itdid-in-two-years-of-the-great-recession/.

18. J. M. Barry, The Great Influenza: The Epic Story of the Deadliest Plague in History (Viking, 2004).

19. O. Dyer, Trump claims public health warnings on COVID-19 are a conspiracy against him. BMJ 368, m941 (2020).

20. C. Funk, A. Tyson, Partisan Differences Over the Pandemic Response Are Growing (Pew Research Center Science and Society, 2020); www.pewresearch.org/science/2020/06/03/ partisan-differences-over-the-pandemic-response-are-growing/.

21. J. Roozenbeek, C. R. Schneider, S. Dryhurst, J. Kerr, A. L. J. Freeman, G. Recchia, A. M. van der Bles, S. van der Linden, Susceptibility to misinformation about COVID-19 around the world. R. Soc. Open Sci. 7, 201199 (2020).

22. A. D. I. Kramer, J. E. Guillory, J. T. Hancock, Experimental evidence of massive-scale emotional contagion through social networks. Proc. Natl. Acad. Sci. 111, 8788-8790 (2014).

23. B. Fischhoff, G. Wong-Parodi, D. R. Garfin, E. A. Holman, R. C. Silver, Public understanding of Ebola risks: Mastering an unfamiliar threat. Risk Anal. 38, 71-83 (2018).

24. J. W. Pennebaker, R. L. Boyd, K. Jordan, K. Blackburn, The Development and Psychometric Properties of LIWC2015 (University of Texas at Austin, 2015).

25. A. Bruns, T. Highfield, J. Burgess, The Arab Spring and social media audiences: English and Arabic Twitter users and their networks. Am. Behav. Sci. 57, 871-898 (2013).

26. H. Carter, J. Drury, G. J. Rubin, R. Williams, R. Amlôt, Applying crowd psychology to develop recommendations for the management of mass decontamination. Health Secur. 13, 45-53 (2015). 
27. P. C. Rosenblatt, R. M. Anderson, P. A. Johnson, The meaning of "Cabin Fever". J. Soc. Psychol. 123, 43-53 (1984).

28. L. E. Smith, B. Duffy, V. Moxham-Hall, L. Strang, S. Wessely, G. J. Rubin, Anger and confrontation during the COVID-19 pandemic: A national cross-sectional survey in the UK. J. R. Soc. Med. 114, 77-90 (2021).

29. T. Schmader, M. Johns, Converging evidence that stereotype threat reduces working memory capacity. J. Pers. Soc. Psychol. 85, 440-452 (2003).

30. K. Klein, A. Boals, The relationship of life event stress and working memory capacity. Appl. Cogn. Psychol. 15, 565-579 (2001).

31. A. Mani, S. Mullainathan, E. Shafir, J. Zhao, Poverty impedes cognitive function. Science 341, 976-980 (2013).

32. S. Seraj, K. G. Blackburn, J. W. Pennebaker, Language left behind on social media exposes the emotional and cognitive costs of a romantic breakup. Proc. Natl. Acad. Sci. 118, e2017154118 (2021).

33. D. Kahneman, Thinking, Fast and Slow (Macmillan, 2011).

34. B. Kleim, A. B. Horn, R. Kraehenmann, M. R. Mehl, A. Ehlers, Early linguistic markers of trauma-specific processing predict post-trauma adjustment. Front. Psych. 9, 645 (2018).

35. A. Boals, J. B. Banks, L. M. Hathaway, D. Schuettler, Coping with stressful events: Use of cognitive words in stressful narratives and the meaning-making process. J. Soc. Clin. Psychol. 30, 378-403 (2011).

36. J. W. Pennebaker, M. R. Mehl, K. G. Niederhoffer, Psychological aspects of natural language use: Our words, our selves. Annu. Rev. Psychol. 54, 547-577 (2003).

37. T. Q. Phan, E. M. Airoldi, A natural experiment of social network formation and dynamics. Proc. Natl. Acad. Sci. 112, 6595-6600 (2015).

38. J. Drury, C. Cocking, S. Reicher, D. Stephen, The nature of collective resilience: Survivor reactions to the 2005 London bombings. Int. J. Mass Emerg. Disasters 27, 66-95 (2009).

39. J. Zaki, Catastrophe compassion: Understanding and extending prosociality under crisis. Trends Cogn. Sci. 24, 587-589 (2020).

40. S. K. Cohn, Pandemics: Waves of disease, waves of hate from the Plague of Athens to A.I.D.S. Hist. J. 85, 535-555 (2012).

41. S. lyengar, G. Sood, Y. Lelkes, Affect, not ideology: A social identity perspective on polarization. Public Opin. Q. 76, 405-431 (2012).

42. W. D. S. Killgore, S. A. Cloonan, E. C. Taylor, D. A. Lucas, N. S. Dailey, Loneliness during the first half-year of COVID-19 lockdowns. Psychiatry Res. 294, 113551 (2020).

43. S. C. Guntuku, G. Sherman, D. C. Stokes, A. K. Agarwal, E. Seltzer, R. M. Merchant, L. H. Ungar, Tracking mental health and symptom mentions on twitter during Covid-19. J. Gen. Intern. Med. 35, 2798-2800 (2020).

44. K. N. Jordan, J. Sterling, J. W. Pennebaker, R. L. Boyd, Examining long-term trends in politics and culture through language of political leaders and cultural institutions. Proc Natl. Acad. Sci. 116, 3476-3481 (2019).

45. J. W. Pennebaker, C. K. Chung, J. Frazee, G. M. Lavergne, D. I. Beaver, When small words foretell academic success: The case of college admissions essays. PLOS ONE 9, e115844 (2014)

46. K. J. Hsu, K. N. Babeva, M. C. Feng, J. F. Hummer, G. C. Davison, Experimentally induced distraction impacts cognitive but not emotional processes in think-aloud cognitive assessment. Front. Psychol. 5, 474 (2014).

47. COVID-19 - Mobility trends reports (Apple, 2020); www.apple.com/covid19/mobility.

48. M. R. Mehl, S. Vazire, N. Ramírez-Esparza, R. B. Slatcher, J. W. Pennebaker, Are women really more talkative than men? Science $\mathbf{3 1 7}, 82-82$ (2007).
49. P. Sharkey, The acute effect of local homicides on children's cognitive performance. Proc. Natl. Acad. Sci. 107, 11733-11738 (2010).

50. A. Gollwitzer, C. Martel, W. J. Brady, P. Pärnamets, I. G. Freedman, E. D. Knowles, J. J. Van Bavel, Partisan differences in physical distancing are linked to health outcomes during the COVID-19 pandemic. Nat. Hum. Behav. 4, 1186-1197 (2020).

51. M. L. Kern, G. Park, J. C. Eichstaedt, H. A. Schwartz, M. Sap, L. K. Smith, L. H. Ungar, Gaining insights from social media language: Methodologies and challenges. Psychol. Methods 21, 507-525 (2016)

52. S. C. Matz, J. J. Gladstone, D. Stillwell, In a world of big data, small effects can still matter: A reply to Boyce, Daly, Hounkpatin, and Wood (2017). Psychol. Sci. 28, 547-550 (2017)

53. M. Kosinski, D. Stillwell, T. Graepel, Private traits and attributes are predictable from digital records of human behavior. Proc. Natl. Acad. Sci. 110, 5802-5805 (2013).

54. G. A. Bonanno, C. R. Brewin, K. Kaniasty, A. M. L. Greca, Weighing the costs of disaster: Consequences, risks, and resilience in individuals, families, and communities. Psychol. Sci. Public Interest 11, 1-49 (2010).

55. P. Whitney, C. A. Rinehart, J. M. Hinson, Framing effects under cognitive load: The role of working memory in risky decisions. Psychon. Bull. Rev. 15, 1179-1184 (2008).

56. Y. Yamada, D.-B. Ćepulić, T. Coll-Martín, S. Debove, G. Gautreau, H. Han, J. Rasmussen, T. P. Tran, G. A. Travaglino; COVIDiSTRESS Global Survey Consortium, A. Lieberoth, COVIDiSTRESS Global Survey dataset on psychological and behavioural consequences of the COVID-19 outbreak. Sci. Data 8, 3 (2021).

57. S. Mannheimer, E. A. Hull, Sharing selves: Developing an ethical framework for curating social media data. Int. J. Digit. Curation 12, 196-209 (2017).

58. M. Zimmer, "But the data is already public": On the ethics of research in Facebook. Ethics Inf. Technol. 12, 313-325 (2010).

59. S. E. Taylor, Tend and befriend: Biobehavioral bases of affiliation under stress. Curr. Dir. Psychol. Sci. 15, 273-277 (2006).

60. W. L. Gardner, S. Gabriel, L. Hochschild, When you and I are "we", you are not threatening The role of self-expansion in social comparison. J. Pers. Soc. Psychol. 82, 239-251 (2002).

Acknowledgments: We are indebted to the following people for advice, data gathering, and/ or feedback on earlier drafts of the paper: A. Kellogg, R. Mallett, S. Macharla, B. Swann, S. Talaifar, and the members of the Pennebaker laboratory. Funding: The current work was funded, in part, by grants from the Templeton Foundation (\#62256), the National Science Foundation (SES1758835), the National Institutes of Health (1R01MH117172), and the Federal Bureau of Investigation (15F06718R0006603), none of which played any role in study design, data collection, or interpretation or in the writing of the report. Author contributions: A.A. and J.W.P. designed the study. A.A. collected and analyzed the data. A.A. and J.W.P. wrote the paper. Competing interests: J.W.P. is the owner of the text analysis program LIWC. The authors declare no other competing interests. Data and materials availability: All the data, scripts, and materials used in this research can be accessed at https://osf.io/3wnuy/.

Submitted 27 January 2021

Accepted 28 July 2021

Published 22 September 2021

$10.1126 /$ sciadv.abg7843

Citation: A. Ashokkumar, J. W. Pennebaker, Social media conversations reveal large psychological shifts caused by COVID-19's onset across U.S. cities. Sci. Adv. 7, eabg7843 (2021). 